\title{
Experiencias del uso de ortoprótesis de mano impresa en 3D (Cyborg Beast) en adolescentes con amputación congénita de mano y sus cuidadores principales: Un estudio de casos
}

\section{Experiences of the use of 3D printed hand ortoprosthesis (Cyborg Beast) in adolescents with congenital hand amputation and their main caregivers: A study of cases}

\section{Carolina Giaconi ${ }^{\mathrm{a}}$, Paula Nahuelhual ${ }^{\mathrm{a}}$, Jacqueline Dote ${ }^{\mathrm{b}}$, Rodrigo Cubillos', Gabriel Fuentes ${ }^{\mathfrak{c}}$, Jorge Zúñiga ${ }^{\mathrm{d}, \mathrm{e}}$}

\author{
aDirección de Investigación y Desarrollo. Teletón Chile \\ bLaboratorio de Órtesis y Prótesis. Teletón Chile \\ unidad de Tecnología Asistiva. Teletón Chile \\ dDepartment of Biomechanics, University of Nebraska Omaha, USA \\ eFacultad de Ciencias de la Salud, Universidad Autónoma de Chile, Chile
}

Recibido: 6 de marzo de 2019; aceptado: 2 de mayo de 2019
Palabras clave: Amputación parcial de mano; ortoprótesis; impresión 3D; rehabilitación; investigación cualitativa 


\section{Abstract}

Objective: To describe the experience of using the 3D-printed prosthetic hand Cyborg Beast in adolescents of Teletón Santiago with congenital hand amputation and their main caregivers who participated in a case study to evaluate the functionality of the prosthetic hand. Clinical Case: Qualitative and descriptive research of case studies using semi-structured interviews with five adolescents with congenital hand amputation and their main caregivers. The information was transcribed and analyzed through open coding. Participants visualize the prosthesis as an opportunity for them by associating it with normality. They also identify positive and negative characteristics, emphasizing in the latter. In addition, they described positive and negative effects produced when using the prosthesis, highlighting that the use of the prosthesis allows them to talk about their condition. Finally, improvement proposals for the prosthesis are presented, defining that the prototype must be corrected and change the age of the target population. Conclusions: The study is the first to investigate a little explored topic, allowing to provide information regarding the subjective experience of adolescents who use a prosthesis that currently has great media importance. The study participants reported difficulties in using the hand prosthesis, either due to materiality and design aspects. The prosthesis did not meet the expectations of use and esthetic.

\section{Keywords:}

Hand partial amputation; prosthetics; 3D printing; rehabilitation; qualitative research

\section{Introducción}

Las amputaciones congénitas de extremidad superior son alteraciones que se producen durante la etapa gestacional $^{1}$, afectando el desarrollo y crecimiento de las extremidades superiores a nivel parcial o completo. Las amputaciones parciales son aquellas en las que existe un segmento remanente de la extremidad, y las completas implican la ausencia del miembro superior ${ }^{2}$. Algunas amputaciones se presentan en el contexto de síndrome y pueden asociarse a otras deficiencias orgánicas a nivel corporal ${ }^{3}$.

La existencia de esta deficiencia requiere un ajuste por parte del niño y/o adolescente a la vida con amputación, siendo la familia un factor crucial ${ }^{4}$, así, factores como depresión, estrés u otros síntomas presentes en miembros del grupo familiar, suelen ser predictores de dificultades para que los niños se adapten a la deficiencia, tengan una buena autoestima y auto concepto ${ }^{5,6}$, lo que afecta directamente su calidad de vida ${ }^{7-9}$. Un estudio que evaluó el auto concepto y el bienestar psicológico, demostró que el auto concepto no era significativamente distinto en grupos de niños con y sin deficien$\operatorname{cias}^{10}$, sin embargo, aquellos niños con anomalías leves -como las amputaciones parciales de mano- tenían peores resultados que aquellos con anomalías severas. También se ha referido que si bien el funcionamiento psicosocial de niños con deficiencias congénitas tiende a no ser distintos a los de niños sin deficiencias?.

Ambos datos son fundamentales a la hora de considerar los tratamientos para las amputaciones congénitas de extremidad superior, pues si bien es una alteración física menor, se ha comprobado que este déficit afecta significativamente el funcionamiento psicosocial y el bienestar. Estas dificultades, son aún más relevantes en la adolescencia, pues los aspectos determinantes en la esfera psicosocial son la imagen corporal, la relación con los pares y el desarrollo de la propia identidad $^{11,12}$.

Por lo anterior, el uso de prótesis es una necesidad para los pacientes con amputaciones ${ }^{13}$, en el caso de niños y adolescentes con amputaciones congénitas de mano, se utilizan de preferencia prótesis pasivas como elemento estético o prótesis activas -mecánicas o eléctricas- que mejoren la funcionalidad ${ }^{14}$. Lo anterior, dependerá del nivel de la amputación y funcionalidad lograda sin prótesis ${ }^{15}$, esta última es fundamental para su adherencia en esta población, ya que se ha reportado baja adherencia al uso de prótesis en pacientes que tienen sensibilidad en el muñón y/o función de agarre ${ }^{16,17}$. La adherencia al uso de prótesis depende de factores como tipo de amputación, edad, género, tipo de prótesis, entre otros ${ }^{15,17,18}$.

\section{Caso Clínico}

El estudio de casos clínicos que se presenta formó parte de una investigación de tipo mixto que tuvo por objetivo evaluar el efecto de la prótesis de mano $\mathrm{Cy}$ borg Beast en la funcionalidad de miembros superiores. Esta prótesis, es una ortoprótesis mecánica de bajo costo desarrollada con tecnología de impresión $3 \mathrm{D}^{19-21}$. En este artículo se presentarán los resultados obtenidos por medio de la metodología cualitativa, a través de la cual se buscó describir las experiencias de cinco adolescentes con amputación parcial congénita de mano y sus cuidadores, respecto del uso de la ortoprótesis de mano impresa en 3-D Cyborg Beast, a partir de un estudio cualitativo de estudio de casos. 
Tabla 1. Características de participantes y sus cuidadores

\begin{tabular}{|c|c|c|c|c|c|c|c|}
\hline \multicolumn{4}{|r|}{ Adolescentes participantes } & \multicolumn{4}{|c|}{ Cuidadores participantes } \\
\hline $\mathrm{N}$ & Edad & Sexo & Diagnóstico & $\mathrm{N}$ & Edad & Sexo & Parentesco con participante \\
\hline P1 & 16 & $\mathrm{H}$ & $\begin{array}{l}\text { Deficiencia congénita mano izqda. Agenesia dedos } \\
\text { mano izqda. }\end{array}$ & C1 & 76 & M & Abuela materna \\
\hline P2 & 14 & M & $\begin{array}{l}\text { Deficiencia congénita de mano izqda. Hipoplasia } \\
\text { Pulgar, deficiencia longitudinal de dedos. }\end{array}$ & $C 2$ & 56 & M & Madre \\
\hline P3 & 16 & $\mathrm{H}$ & $\begin{array}{l}\text { Deficiencia parcial de mano Izquierda con } \\
\text { remanente de meñique y pulgar cortos }\end{array}$ & C3 & 37 & M & Madre \\
\hline P4 & 14 & M & Agenesia mano izqda. & C4 & 52 & M & Madre \\
\hline P5 & 19 & $\mathrm{H}$ & Síndrome de Poland. & C5 & 52 & M & Madre \\
\hline
\end{tabular}

H: hombre; M: mujer; P: participante; C: cuidador

\section{Participantes}

Los participantes fueron cinco adolescentes con amputación parcial de mano de origen congénito del Instituto Teletón Santiago y sus cuidadores principales (tabla 1). Se incluyeron usuarios con amputación congénita de mano (izquierda o derecha) de 12 a 17 años de edad y que residían en Santiago. Todos ellos tenían características físicas similares en relación al tipo de amputación. Se incluyeron cinco pacientes pues se contó con cinco prototipos de la ortoprótesis, las fueron donadas a Teletón. Cada una fue diseñada y personalizada para cada usuario, según preferencia de colores y características físicas de su mano.

\section{Producción y análisis de datos}

Se realizaron diez entrevistas semiestructuradas una vez finalizado el periodo de uso de la prótesis (cuatro meses). Las entrevistas se realizaron en el Instituto Teletón Santiago y fueron grabadas, en cada oportunidad se citó al adolescente y a su cuidador principal, a ambos se les explicó en qué consistiría la entrevista y se dio paso entrevistarlos individualmente. Para la producción de datos, se contó con dos guías de entrevista, una para adolescentes y otra para cuidadores, las que incluyeron preguntas sobre: primera impresión, funciones, impacto social y elección de uso, y significados relacionados al uso de la ortoprótesis. Para el análisis, se realizó un análisis de contenido cualitativo a través de codificación abierta ${ }^{22}$, primero se transcribieron los audios de las entrevistas y posteriormente, se analizaron línea a línea asignando conceptos de manera inductiva.

\section{Consideraciones éticas}

Este trabajo fue aprobado por el Comité Ético Científico de la Sociedad Pro Ayuda del Niño Lisado (Proyecto $n^{\circ}$ 43/2014). Los participantes firmaron asentimiento y consentimiento informado.

\section{Resultados}

A partir del análisis se construyeron cinco categorías generales respecto de la experiencia del uso de la ortoprótesis Cyborg Beast (figura 1):

\section{a. Significados atribuidos a la mano Cyborg Beast}

Los adolescentes y sus cuidadores, conciben la mano como una oportunidad, debido a que se sienten beneficiados al poder participar del estudio y evaluación de la ortoprótesis, y, además, porque creen que la mano es una oportunidad que permitirá crear mejores prototipos para los niños y jóvenes que tienen una amputación. En palabras de una cuidadora "puede que no sea lo que uno esperaba, pero yo digo a lo mejor con esto

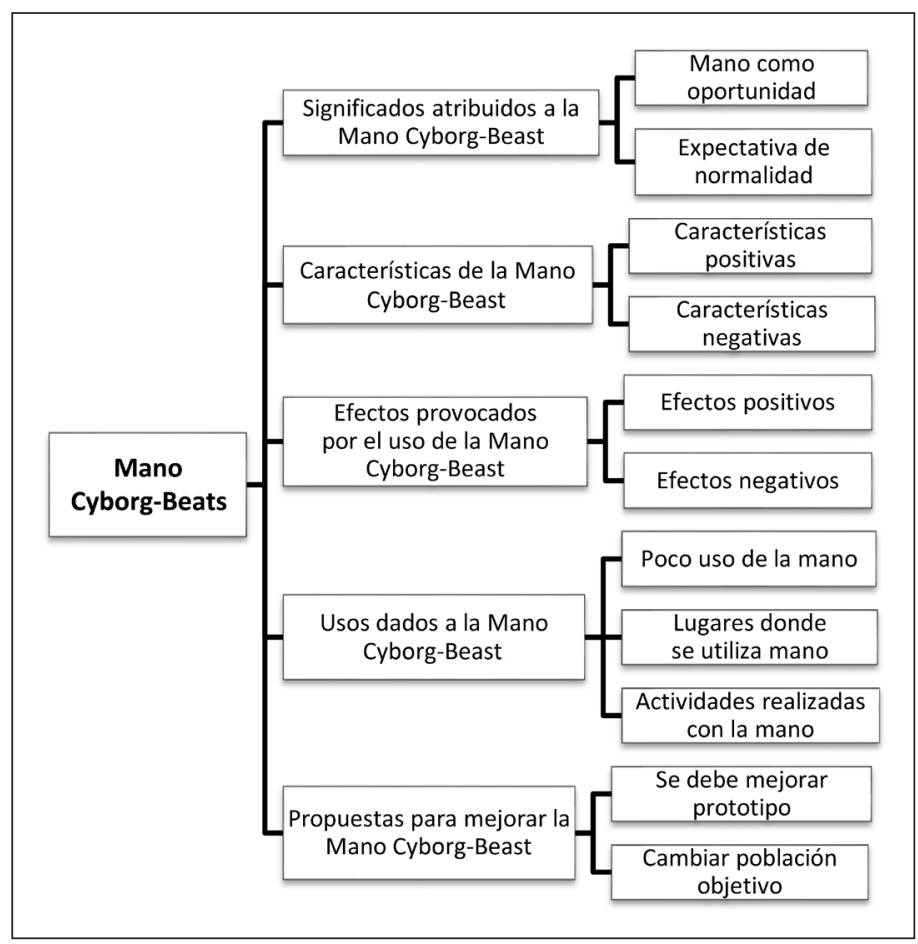

Figura 1. Esquema de organización jerárquica de categorías y subcategorías. 
van a... en el tiempo van a hacer otra' cosa' entonce' uno nunca puede negarse a... a experimentar algo que puede en algún momento que le sirva realmente... que le haga un aporte en su diario vivir" (C2).

Los adolescentes significan el uso de la mano con expectativas de normalidad, indicando que se sienten completos al usar esta prótesis, lo anterior, queda de manifiesto a través de lo referido por uno de los adolescentes "de verse con, con los cinco [dedos] ya, eso marca la diferencia (...) yo por lo menos que tengo esta enfermedad de chico siempre como que, que pensaba, eh, cómo sería y cómo me vería con los cinco dedos" (P5).

\section{b. Características de la mano Cyborg-Beast}

Los participantes reconocen que la ortoprótesis tiene características positivas y negativas. Como característica positiva, reconocen que es fácil de arreglar cuando presenta desperfectos. En relación a lo negativo, adolescentes y cuidadores identifican que la prótesis es frágil, razón por la que los usuarios tienen miedo al usarla, pues puede romperse fácilmente, “pa' usar la mano me dijeron que... que apretara pa'ver la fuerza $y$ apreté y este el, el que modera la, la presión se salió así que por eso no la ocupo mucho ya que se puede salir $y$ romper" (P3).

Ambos grupos identifican que la mano es poco funcional, pues es menos funcional que su mano real y soporta poco peso, no cumpliendo con las expectativas que ellos tenían al iniciar su uso, "lo más pesado que agarré fue un vaso con agua (...) creo que era la mía que se soltaba todo el cosito, entonces era muy, también era mucho más difícil por eso (P5).

Finalmente, cuidadores y adolescentes identifican que la prótesis tiene problemas estéticos, pues es muy grande, llamativa, tosca, masculina y robótica, lo que provoca dificultades para su uso en los adolescentes. En palabra de una cuidadora, "la encontré rara. Por toda la, los pernos, tooodo. Pensé que no, no se iba a ver tanto, pero... no sé, es como rara. La encontré, no es fea, pero tampoco es bonita, yo no la usaría en la calle" (C2). A lo anterior, agregan los cuidadores que estas características harían que la prótesis fuese más difícil de utilizar en mujeres.

\section{c. Efectos provocados por uso de la mano Cyborg-Beast}

El uso de la prótesis, según los participantes, causa efectos positivos y negativos. Como efectos positivos, los adolescentes indican que la prótesis les ha permitido hablar de su discapacidad con otras personas, "uno se siente más cómodo, ya que al, al hablar con tu' amigo' y decir: “ioye, me llegó esta mano!”, te dicen: “joh!, qué bakán, anda a mostrárnola y uno puede tocar el tema que ante' no se tocaba ya que, o da vergüenza, o que uno lo podian molestar" (P3).
En cuanto a los efectos negativos, adolescentes y cuidadores, refieren que el uso constante de la prótesis les provoca lesiones, pues el material de confección es de un plástico "muy duro". Además, agregan que su uso provoca molestias, pues es sentida como un objeto ajeno al cuerpo, "como que no siento que es parte de mí porque (...) es como algo que siento incómodo encima de la mano" (P4).

Finalmente, existe cierta incoherencia en el discurso de los participantes, pues ambos grupos identifican que, si bien la prótesis disminuye la dificultad para realizar ciertas actividades, provoca dificultades para desarrollar otras acciones. Lo anterior puede visualizarse en el discurso de uno de los adolescentes "limitaba el movimiento, a mí me lo limitaba, lo veo la mano de [nombra a compañero] y a él, él si obviamente no tenía tanto dedo como yo, entonces a él si le ayuda, entonces a mí por ejemplo me limitaba un poquito más en hacer cosas, en agarrar, para tocar la guitarra, por ejemplo" (P5).

\section{d. Usos de la mano Cyborg Beast}

Los participantes refieren que utilizaron poco las prótesis. Como razones de este poco uso, los adolescentes indicaron que no les gustó utilizarla, mientras que sus cuidadores, refirieron que los adolescentes tuvieron dificultades para adaptarse a ella porque no se adaptó a sus necesidades. Tanto adolescentes como cuidadores, agregaron que la prótesis nunca fue utilizada en los colegios y que sólo se ocupó en los hogares. Ambos grupos también agregaron que quienes aceptan su condición prefieren no utilizar una prótesis, "es que he estado quince años con mi mano normal, entonce' $a$, que me den una mano nueva es como... igual un poco choqueante... yo prefiero mi mano normal la verdá (...) siendo sincera, bastante honesta, prefiero mi mano normal (...) la verdá. me siento..., no sé conforme conmigo misma" (P2). Este discurso permite dar cuenta de que los participantes de este estudio estaban habituados al uso de su mano con la funcionalidad que ésta ya les permitía, por lo que el uso de la ortoprótesis no consistió en un cambio significativo para ellos. En relación a los usos dados a la prótesis, los adolescentes indicaron que la principal actividad que realizaron fue tomar objetos, los cuidadores agregan que los adolescentes utilizaron la mano para jugar o divertirse con sus familiares.

\section{e. Propuestas para mejorar la mano Cyborg Beast}

Finalmente, los participantes indicaron propuestas para mejorar la ortoprótesis Cyborg Beast. En primer lugar, indicaron que debe mejorarse el prototipo, aumentando la variedad de colores, agregando colores poco llamativos y parecidos a la "piel", "tendría más variedad de color (...) porque a mí me dijeron que había solamente rosado (...) también haría como... piel, claro, 
sí, colore' ma' neutro en sí, no tan llamativo" (P2). Ambos grupos agregan que se debe mejorar la adherencia de las siliconas protectoras en los extremos distales de los dedos de la ortoprótesis y que ésta debería hacerse más parecida a una mano real.

Los adolescentes agregan que la prótesis debería tener movimiento independiente de los dedos, "intentar mover los deo' porque son así movimiento como, como una mano normal (...) que sea como una mano de verdad, pero, de plástico" (P1). Todas estas mejoras harían que la prótesis fuera más parecida a una mano real, provocando un aumento de su uso.

Por último, los participantes concuerdan en que se debe cambiar la población objetivo de la prótesis, debiendo entregarse la mano Cyborg Beast a la población infantil, puesto que los niños podrían adaptarse con mayor facilidad que los adolescentes "yo creo que, a los siete, ocho (...), porque como a esa edá a ello' no les importa tanto y... como cuando se es más chico no, no hacen tanto bullying" (C2).

\section{Discusión}

Los resultados del estudio dan cuenta que los adolescentes utilizaron mínimamente la ortoprótesis de mano, pues tuvieron serias dificultades para acostumbrarse a ella, por estar adaptados a su déficit, o bien, porque la prótesis fue percibida como un objeto extraño a su cuerpo, que además era demasiado llamativa, por lo que no se condecía con su expectativa de "normalidad". Respecto de la funcionalidad manual, los pacientes que participaron en este estudio, habían resuelto la mayoría de sus actividades con el remanente de mano que tenían, por ello, describen que el mayor problema que ven a la prótesis es el estético, ya que ellos han buscado no seguir llamando la atención de su entorno con la prótesis, dado que sienten que antes ya lo habían hecho con la amputación. Lo que más repitieron los adolescentes participantes es la necesidad de que la prótesis fuese lo más parecida a una "mano normal", lo que se relaciona con los estudios que han evaluado el autoconcepto en niños con deficiencias leves ${ }^{10}$.

Es importante destacar que los participantes relataron dificultad para acostumbrarse a la mano protésica, lo que puede explicarse debido a que durante la adolescencia aumenta la preocupación por el aspecto corporal, lo que genera una gran preocupación en los adolescentes con extrañamiento y rechazo del propio cuerpo, inseguridad respecto a su atractivo, y aumento de interés por la sexualidad ${ }^{23}$. Lo anterior es relevante particularmente en el caso de los adolescentes con deficiencias visibles, por lo que incorporar una prótesis durante la adolescencia puede ser contraproducente ${ }^{4,9}$, pues como se indicó, los adolescentes declaran haberse acostumbrado a "no tener mano".

Lo anterior, es reforzado por los participantes al coincidir en que sería bueno probar este tipo de prótesis en niños más pequeños, ya que la aceptación por parte de este grupo etario consideran, podría ser mejor, pues ellos no estarían acostumbrados a su amputación y por su naturaleza de niños, podrían ver la prótesis como un juguete, pudiéndola incorporar más fácilmente a su vida diaria.

Los resultados de este estudio, relevan la importancia de incorporar las expectativas de los participantes en la evaluación de prótesis o cualquier ayuda técnica, muchas veces desarrolladas sólo desde el punto de vista del diseño e ingeniería, siendo fundamental considerar también la etapa del ciclo vital que los pacientes están viviendo. Finalmente, respecto de las altas expectativas de uso de la prótesis, se cree que la alta difusión mediática realizada en medios de comunicación producto de la innovación que implica la prótesis, pudo generar mayores expectativas entre los participantes, por lo que se cree fundamental seguir desarrollando estudios de funcionalidad y de significados, con el fin de generar evidencia que pueda apoyar la toma de decisiones y la entrega de información empírica respecto de estas innovaciones.

\section{Conclusiones}

El presente estudio permite evidenciar la necesidad de considerar la visión y subjetividad de los usuarios que harán uso de estas ortprótesis, pues la adaptación a ellas siempre será una experiencia individual, que variará de acuerdo a distintos ámbitos, como la funcionalidad lograda con remanente de mano, edad, adaptación a la condición y contexto sociocultural; todos elementos descritos por los participantes. La ortoprótesis no cumplió las expectativas de uso y estética.

\section{Responsabilidades Éticas}

Protección de personas y animales: Los autores declaran que los procedimientos seguidos se conformaron a las normas éticas del comité de experimentación humana responsable y de acuerdo con la Asociación Médica Mundial y la Declaración de Helsinki.

Confidencialidad de los datos: Los autores declaran que han seguido los protocolos de su centro de trabajo sobre la publicación de datos de pacientes. 
Derecho a la privacidad y consentimiento informado: Los autores han obtenido el consentimiento informado de los pacientes y/o sujetos referidos en el artículo. Este documento obra en poder del autor de correspondencia.

\section{Conflicto de intereses}

Jorge Zúñiga es el diseñador de la mano protésica Cyborg Beast. El resto de los investigadores declara no tener conflicto de interés.

\section{Referencias}

1. Dy CJ, Swarup I, Daluiski A. Embryology, diagnosis, and evaluation of congenital hand anomalies. Current reviews in musculoskeletal medicine. 2014;7(1):60-7.

2. Kozin SH. Upper-extremity congenital anomalies. The Journal of Bone \& Joint Surgery. 2003;85(8):1564-76.

3. Bourke G. Congenital hand anomalies. Orthopaedics and Trauma. 2011;25(2):143-54. doi: 10.1016/j. mporth.2010.10.006.

4. Tyc VL. Psychosocial adaptation of children and adolescents with limb deficiencies: A review. Clinical Psychology Review. 1992;12(3):275-91.

5. Varni JW, Rubenfeld LA, Talbot D, Setoguchi Y. Family functioning, temperament, and psychologic adaptation in children with congenital or acquired limb deficiencies. Pediatrics. 1989;84(2):323-30.

6. Varni JW, Setoguchi Y, Rappaport LR, Talbot D. Psychological adjustment and perceived social support in children with congenital/acquired limb deficiencies. Journal of behavioral medicine. 1992;15(1):31-44.

7. Didierjean-Pillet A, editor. [Psychological approach to congenital hand deformities. Congenital deformities, the desire to know]. Annales de chirurgie plastique et esthetique; 2002.

8. Hermansson L, Eliasson A, Engström I. Psychosocial adjustment in Swedish children with upper-limb reduction deficiency and a myoelectric prosthetic hand. Acta Paediatrica. 2005;94(4):47988 .
9. Michielsen A, Van Wijk I, Ketelaar M. Participation and quality of life in children and adolescents with congenital limb deficiencies: A narrative review. Prosthet Orthot Int. 2010;34(4):351-61. doi: 10.3109/03093646.2010.495371. PubMed PMID: 20704518

10. Andersson G-B, Gillberg C, Fernell E, Johansson M, Nachemson A. Children with surgically corrected hand deformities and upper limb deficiencies: self-concept and psychological well-being. Journal of Hand Surgery (European Volume). 2011;36(9):795-801.

11. Garrett JF, Levine ES. Psychological practices with the physically disabled 1962.

12. Gaete VJRcdp. Desarrollo psicosocial del adolescente2015;86(6):436-43.

13. James MA, Bagley AM, Brasington $\mathrm{K}$, Lutz C, McConnell S, Molitor F. Impact of prostheses on function and quality of life for children with unilateral congenital below-the-elbow deficiency. J Bone Joint Surg Am. 2006;88(11):2356-65. doi: 10.2106/JBJS.E.01146. PubMed PMID: 17079391.

14. Shaperman J, Landsberger SE, Setoguchi Y. Early upper limb prosthesis fitting: when and what do we fit. JPO: Journal of Prosthetics and Orthotics. 2003;15(1):117.

15. Biddiss E, Chau T. The roles of predisposing characteristics, established need, and enabling resources on upper extremity prosthesis use and abandonment. Disability and Rehabilitation: Assistive Technology. 2007;2(2):71-84

16. Biddiss E, Chau T. Upper-limb prosthetics: critical factors in device abandonment. American journal of physical medicine \& rehabilitation. 2007;86(12):977-87.

17. Biddiss EA, Chau TT. Upper limb prosthesis use and abandonment: a survey of the last 25 years. Prosthetics and orthotics international. 2007;31(3):23657.

18. Biddiss E, Beaton D, Chau T. Consumer design priorities for upper limb prosthetics. Disability and Rehabilitation: Assistive Technology. 2007;2(6):346-57.

19. Zuniga J, Katsavelis D, Peck J, Stollberg J, Petrykowski M, Carson A, Fernandez C. Cyborg beast: a low-cost 3d-printed prosthetic hand for children with upperlimb differences. BMC research notes. 2015;8(1):1-9.

20. Rengier F, Mehndiratta A, von Tengg-Kobligk H, Zechmann CM, Unterhinninghofen R, Kauczor $\mathrm{H}-\mathrm{U}$, Giesel FL. 3D printing based on imaging data: review of medical applications. International journal of computer assisted radiology and surgery. 2010;5(4):335-41.

21. Tanaka KS, Lightdale-Miric N. Advances in 3D-Printed Pediatric Prostheses for Upper Extremity Differences. J Bone Joint Surg Am. 2016;98(15):1320-6.

22. Strauss AL, Corbin J. Bases de la investigación cualitativa: técnicas y procedimientos para desarrollar la teoría fundamentada: Universidad de Antioquia Medellín; 2002.

23. Radzik M. Psychosocial development in normal adolescents. Adolescent health care: A practical guide2008. p. 27-31. 